\title{
Non-Existence of Spontaneous Magnetization in a One-Dimensional Ising Ferromagnet
}

\author{
Freeman J. Dyson \\ Institute for Advanced Study, Princeton, New Jersey \\ Received January 13, 1969
}

Abstract. It is proved that an infinite linear chain of spins $\mu_{s}= \pm 1$, with an interaction energy

$$
H=-\Sigma J(i-j) \mu_{i} \mu_{j},
$$

has zero spontaneous magnetization at all finite temperatures, provided that $J(n)$ is non-negative and that

$$
(\log \log N)^{-1} \sum_{1}^{n} n J(n) \rightarrow 0 \quad \text { as } \quad N \rightarrow \infty .
$$

This shows that a theorem of Ruelue, establishing the absence of long-range order when the sum $\sum n J(n)$ converges, is not the best possible.

\section{Result}

This paper is a sequel to an earlier one [1] dealing with the existence of phase-transitions in the infinite Ising ferromagnet with energy

$$
H=-\sum_{i>j} J(i-j) \mu_{i} \mu_{j}
$$

In [1] it was proved that a transition at a finite temperature from zero to nonzero spontaneous magnetization does occur if $J(n)$ is positive and monotonically decreasing and if

$$
\begin{aligned}
& M_{0}=\sum_{n=1}^{\infty} J(n)<\infty, \\
& K_{3}^{\prime}=\sum_{n=1}^{\infty}(\log \log (n+4))\left[n^{3} J(n)\right]^{-1}<\infty .
\end{aligned}
$$

On the other hand, Ruelle [2] has proved that if $J(n)$ is positive and

$$
M_{1}=\sum_{n=1}^{\infty} n J(n)<\infty
$$

then there is zero spontaneous magnetization at all temperatures. A gap remains between the conditions (1.3) and (1.4), including the particularly interesting case

$$
J(n)=n^{-2} .
$$


Within the gap the existence of spontaneous magnetization is still in doubt. KAC and THompson [3] conjectured that (1.4) would be necessary as well as sufficient for the non-existence of spontaneous magnetization. In this paper we narrow the gap very slightly, not enough to deal with the case (1.5), but enough to exclude the Kac-Thompson conjecture.

Theorem. In the infinite Ising ferromagnet with energy (1.1), there is zero spontaneous magnetization at all finite temperatures provided that $J(n)$ is non-negative and

$$
(\log \log N)^{-1} \sum_{n=1}^{N} n J(n) \rightarrow 0 \quad \text { as } \quad N \rightarrow \infty .
$$

\section{Proof}

The proof of the theorem is similar to the proof of Theorem 6 in [1], and is entirely based on the work of GRIFFITHS [4]. The same idea which was applied to the "Hierarchical Model" in the proof of Theorem 6 is now applied directly to the linear model (1.1).

We denote by $L_{0}$ the Ising ferromagnet with the energy (1.1). For any positive integer $p$ we define an Ising ferromagnet $L_{p}$ which is obtained by locking together blocks of $2^{p}$ consecutive spins in $L_{0}$. Equivalently, $L_{p}$ is obtained from $L_{p-1}$ by locking together pairs of neighbouring spins. A single spin $\mu_{j}$ in $L_{p}$ replaces a block of spins $\mu_{k}$ in $L_{0}$ with

$$
(j-1) 2^{p}+1 \leqq k \leqq j 2^{n} .
$$

Therefore the model $L_{p}$ has the energy

with

$$
H_{p}=-\sum_{i>j} J_{p}(i-j) \mu_{i} \mu_{j}
$$

$$
J_{p}(n)=\sum_{k=-2^{p}}^{2^{p}}\left[2^{p}-|k|\right] J\left(n .2^{p}+k\right) .
$$

The sum (1.2) calculated for the model $L_{p}$ is

$$
M_{p, 0}=\sum_{n=1}^{\infty} J_{p}(n)=\sum_{k=1}^{\infty} J(k) \operatorname{Min}\left[k, 2^{p}\right] .
$$

The condition (1.6) implies that $M_{0}$ given by (1.2) and all the $M_{p, 0}$ given by (2.4) converge. Therefore the theorem of GaLlavotri and MiracLe-SoLe [5] ensures that the models $L_{p}$ are well-defined thermodynamic systems.

Let long-range order in the model $L_{p}$ be measured by the coefficient

$$
g_{p}(k)=k^{-2}\left\langle\left(\sum_{j=1}^{k} \mu_{j}\right)^{2}\right\rangle_{p}
$$


the average being taken in $L_{p}$ at some fixed temperature $T$. The spontaneous magnetization $m_{p}$ of $L_{p}$ is then given by

$$
m_{p}^{2}=\operatorname{Lim}_{k \rightarrow \infty} g_{p}(k), \quad 0 \leqq m_{p} \leqq 1 .
$$

The limit exists according to an argument of Griffiths [4]. Let now $P_{p}$ be the probability that two neighbouring spins are parallel in the model $L_{p}$. From (2.5) we deduce

$$
g_{p}(2 k)=(2 k)^{-2} \sum_{j=1}^{k} P_{p}\left\langle 2 \mu_{2 j}\left(\sum_{i=1}^{2 k} \mu_{i}\right)\right\rangle_{p, L},
$$

where the suffix $L$ means that the spins $\mu_{2 j-1}$ and $\mu_{2 j}$ are to be locked together in $L_{p}$ while taking the average. By GRIfFITHs [4], the average in (2.7) can only increase if all neighbouring spin-pairs are locked together, thus converting the model $L_{p}$ into $L_{p+1}$. Thus (2.7) implies

$$
g_{p}(2 k) \leqq P_{p} g_{p+1}(k) .
$$

Letting $k \rightarrow \infty$ according to (2.6),

and therefore

$$
m_{p}^{2} \leqq P_{p} m_{p+1}^{2},
$$

$$
m_{0}^{2} \leqq \prod_{p=0}^{\infty} P_{p} .
$$

Since $m_{0}$ is the spontaneous magnetization of the model (1.1), the theorem is proved if we can show that the product on the right of (2.10) diverges to zero.

An upper bound to $P_{p}$ is obtained from the theorem of GRIFFitis [4] which states that the probability for the spins $\left(\mu, \mu^{\prime}\right)$ to be parallel is increased if all the remaining spins are locked in an orientation parallel to $\mu^{\prime}$. We thus find

$$
P_{p} \leqq\left[1+\exp \left(-4 \beta M_{p, 0}\right)\right]^{-1},
$$

with $M_{p, 0}$ given by (2.4). Hence $m_{0}=0$ provided that the series

$$
S=\sum_{p=0}^{\infty} \exp \left(-4 \beta M_{p, 0}\right)
$$

diverges. Now (1.6) implies that for every $\varepsilon>0$ and all sufficiently large $p$

$$
\sum_{n=1}^{2^{p}} n J(n)<\varepsilon \log p
$$


Therefore (2.4) gives for all large $p$

$$
\begin{aligned}
M_{p, 0} & =\sum_{n=1}^{2^{p}} n J(n)+\sum_{q=p}^{\infty} \sum_{n=2^{q}+1}^{2^{q+1}} 2^{p} J(n) \\
& \leqq \sum_{n=1}^{2^{p}} n J(n)+\sum_{q=p}^{\infty} 2^{p-q} \sum_{n=1}^{2^{q+1}} n J(n) \\
& <\varepsilon \log p+\sum_{q=p}^{\infty} 2^{p-q} \varepsilon \log (1+q) \\
& <4 \varepsilon \log p .
\end{aligned}
$$

Hence the terms of the series (2.12) satisfy

$$
\exp \left(-4 \beta M_{p, 0}\right)>p^{-16 \varepsilon \beta}, \quad p>p_{0}(\varepsilon) .
$$

Choosing $\varepsilon=(16 \beta)^{-1}$, the series diverges and the theorem is thereby proved.

\section{Addendum}

References to two earlier papers, Fisher [6] and GrIfFiths [7], ought to have been included in my paper [1]. I am grateful to the authors for bringing these papers to my attention. Fisher [6] is relevant to my work in two respects. Firstly, FISHER studies a one-dimensional spin-system with long-range interactions, solves it exactly, and proves that under suitable conditions a phase-transition exists. He carries through this beautiful and complete analysis for a model which is at least as "realistic" as my hierarchical model. Secondly, Fisher states explicitly the conjecture which appears as Corollary 1 to Theorem 1 in my paper [1], and attributes this conjecture to $\mathrm{KAC}_{\mathrm{AC}}$ [8]. GRIFFITHS [7] has greatly clarified the interrelations between the various alternative definitions of "spontaneous magnetization" in an Ising ferromagnet. I regret that in writing my paper [1] I did not make use of GRIFFITHs' nomenclature, and I urge anybody writing on this subject in future to do so.

\section{References}

1. Dyson, F. J.: Existence of a phase-transition in a one-dimensional Ising ferromagnet. Commun. Math. Phys. (to appear).

2. Ruelle, D.: Commun. Math. Phys. 9, 267 (1968).

3. KAC, M., and C. J. Thompson: Critical behavior of several lattice models with long-range interactions. Preprint, Rockefeller University, 1968.

4. Griffiths, R. B.: J. Math. Phys. 8, 478 (1967).

5. Gallavotti, G., and S. Miracle-Sole: Commun. Math. Phys. 5, 317 (1967).

6. Fisher, M. E.: Physics 3, 255 (1967).

7. Griffiths, R. B.: Phys. Rev. 152, 240 (1966).

8. Kac, M.: Remark at the Conference on Phase Transitions at Brown University. Providence, R. I. June 1962. 